\title{
A Study on the Application of Micro Blog Platform in Contemporary College English Teaching
}

\author{
Ling Luo ${ }^{1, a}$ \\ ${ }^{1}$ Neijiang Normal University, Neijiang City, Sichuan Province, 641000, China
}

\section{Keywords: Micro Blog; College English; Application; Feasibility; Countermeasures}

\begin{abstract}
The rapid development of network technology has a great impact on students' learning. In particular, the emergence of blogs, wikis, podcasts and other application software based on web technology provide a broad platform for sharing resources and information, and shorten the distance between people. Undoubtedly, this will create better conditions for college English learning. The function of Micro Blog coincides with the principle of contemporary constructivism. Its application in English teaching provides a new way to promote students' English learning, especially the independent learning. Based on the author's learning and practical experience, this paper first analyzed the characteristics of Micro Blog, and then discussed the feasibility of the application of Micro Blog in college English teaching. Finally, this paper put forward measures to promote the construction of Micro Blog platform of college English teaching.
\end{abstract}

\section{Introduction}

With the emergence of Web 2. 0 technology, the network quickly changes from the static information medium to a global communication platform. Blogs, wikis, and podcasts whose content is written by the user are favored by more people, especially college scholars. How to use the language interactive platform of Micro Blog to improve students' comprehensive proficiency in applying English has become a research topic, which discusses how to improve the quality of English teaching in network environment. Based on the constructivist learning theory, this paper studies the function and advantage of Micro Blog in the English knowledge construction.

\section{The Characteristics of Micro Blog}

Micro Blog is an information sharing, dissemination and access platform based on the user relationship. Users can set up a personal community with the use of WEB, WAP and a variety of clients, and update the information within 140 words to achieve the real-time sharing. With the rapid development of Micro Blog in China, it has become one of the most popular network applications currently. If the phone is the hardware for everyone to communicate with others at any time in the modern society, Micro Blog is the software for people to communicate with others at any time. Compared with other social media, Micro Blog has the following characteristics:

First, it is simple and convenient. The content in Micro Blog can be a simple sentence, a picture and an emoticon. As long as people click the mouse or conduct the simple button operation, they can release their content. Second, it is timely and rapid. With the support of mobile devices, users can release Micro Blog information anytime and anywhere in the first time or search the latest information in every corner of the world. At the same time, the forwarding function of Micro Blog can make the information dissemination more rapid. Third, it is extensive and open. There exists no central leader in Micro Blog like the traditional communication tools and it advocates equal exchanges with no center. It has attracted users to participate extensively and everyone is a recorder and publisher in Micro Blog. Users can choose to follow others, receive their messages, comment these messages and forward them. Users can express their ideas and views freely, which will achieve an efficient sharing of information. Fourth, it is rich and diverse. Users can publish texts, pictures, audios, videos and other multimedia information. The content form is diverse to give the user a 
variety of sensory stimulus. Moreover, the distribution way can also be diversified. The user can use mobile phones, computers and other terminals to browse the Micro Blog. Even the text message can become a way to browse Micro Blog. Because of the above advantages of Micro Blog, it has been widely used in news communication, business, library, education, politics and other fields, which has brought great convenience for people's lives, learning and work.

\section{The Feasibility of the Application of Micro Blog in College English Teaching}

Material conditions have been popularized. With the continuous development of social economy, communication technology and electronic technology, and with the decrease of communication and network charges, mobile phones, computers and networks have been basically popular in college students, which provides material conditions for the application of Micro Blog in college English teaching.

Micro Blog caters to college students' needs. Nowadays, many college students have develop a habit of bringing the phone all the time. Whether it is in the rest of the class, in the cafeteria or in the bus stop, the mobile phone has become their essential partner. Timeliness, richness, openness and diversity of Micro Blog deeply attract students. They are proficient in using mobile phones and other communication tools. If we use Micro Blog which students have strong dependence on to teach college English, we will promote the teaching and learning.

It conforms to the College English Teaching Requirement. College English Teaching Requirement (2007 edition) clearly points out that "the colleges and universities should make full use of modern information technology and English teaching model based on computer and classroom to improve the teaching model with the teacher as the center. The new model should be supported by modern information technology, especially network technology, so that the teaching and learning of English cannot be limited to the time and place, so as to develop individual and independent learning." There is no doubt that the cross-time and space feature and diversity of Micro Blog can make up for the shortcomings of traditional teaching. It is conducive to students' individual and independent learning, which can achieve mobile learning and ubiquitous learning.

\section{Measures to Promote the Construction of Micro Blog Platform of College English Teaching}

To attach importance to the use of new media tools in college English teaching. The use of new media to carry out college English learning can integrate learning time, so that students can use the fragmented time to accumulate English knowledge to absorb English knowledge and skills anytime and anywhere; combining learning and entertainment will greatly improve students' learning interest and learning efficiency; students can learning English based on their individual needs and acceptance ability, which will avoid the lack of individual learning in traditional college English teaching. In order to change the old ideas and views, teachers should overcome the old concept at the first place and they should not stick to convention. Second, they should not lose confidence in innovation because of their ability. Schools should provide opportunities and build a platform for young teachers to innovate education. Third, schools should find the starting point and the entry point of the innovation. It is necessary to keep up with the development of the times. However, it cannot be too advanced. Teachers should pay attention to the use of new media and establish a vision for the future development. In the Micro Blog English teaching, teachers should actively explore the teaching potential of WeChat, APP and other advanced media, so as to maximize the use of advanced technology to disseminate cultural knowledge.

To strengthen the top-level design of Micro Blog college English teaching. Based on the current educational situation in China, to build a new media platform of English teaching, schools must have a scientific and reasonable work plan with the support of leaders. First is to identify the positioning and take the new media platform as a supplementary platform for classroom teaching. This platform should set up test, practice, culture, entertainment and other columns to enhance the 
attraction of the platform. At the same time, schools should avoid the interference of the auxiliary platform to the classroom teaching platform. We should avoid the phenomenon that students use phones in classroom teaching. Second, schools must scientifically design the Micro Blog system and take the English learning as a common goal. The system should combine college English teachers Micro Blog team, college students Micro Blog users and the existing English Micro Blog organically, so as to build a multilevel Micro Blog English learning network. The third is to support the construction of new media teaching platform and the implementation of new media education reform project with real input in English teaching innovation. Fourth, schools must rationally encourage the construction of the innovative platform. With the combination of basic quantitative evaluation and subjective effect evaluation, the new media teaching work should be included in the workload of teachers. The fifth is to form a certain scale. Schools should connect the college English teachers in all universities with the use of the English Micro Blog to build a Micro Blog team of college English teacher, so that it will play the scale effect with new media education flourishing.

To strengthen the construction of Micro Blog team of college English teaching. The establishment of a Micro Blog team of English teaching with college English teachers as the main force is the key to build a new media innovative platform. First is to form a reasonable division of labor. With the use of questionnaire survey, teachers can understand students' interest in learning college English in Micro Blog, so as to set the division and direction of Micro Blog team of college English teacher. Second, it is necessary to enhance teachers' ability. College English teachers are proficient in language, but they are not competent in new media technology. Therefore, they need to strengthen capacity training, so that teachers can have an ability in using Micro Blog. Third, schools must carry out external exchanges. They should organize interaction and cooperation between colleges and universities, or universities and new media professional platform; they should draw experience on the new media platform construction and activities. Fourth, schools should support the development of student-related associations and integrate associations of new media or student college English learning. They can carry out online and offline activities to promote emotional communication with fans in Micro Blog platform of college English.

To strengthen the cooperation with social English Micro Blog accounts. There is a large number of English communicators and English learners in Micro Blog platform. Some are excellent and responsible communicators, but some are very poor. There exist about more than 40 English Micro Blog accounts with more than five hundred thousand fans in Sina Micro Blog. Accounts with over one million fans are about twenty. The representative ones include "Sina Daily English Recommendation", "English Prince-Zhang Jieying", "English Sentence", "Pocket English" and "popular English". The existing English Micro Blog can be divided into the class of famous and beautiful English sentence, the class of spoken expression, the class of audio and video recommendation, the class of test skills and the comprehensive class. Among them, there are many accounts with the class of famous and beautiful English sentence. These sentences are quotes by famous people, English prose and novels, movie lines and catchwords, but such Micro Blog accounts often have grammatical errors, translation errors and misspellings. In addition, there are some Micro Blog accounts which release various types of advertising, jokes, constellation and fortune-telling content in the name of English learning in the long term. This disturbs the majority of English learners. Schools can organize college English teachers to study English Micro Blog accounts with much attention to analyze their characteristics. Then, they can summarize the pros and cons of existing college English Micro Blog accounts and strengthen cooperation with them consciously. This will not only lay a foundation for the optimization of the Micro Blog accounts of college English teachers, but also can integrate resources to form a joint force of the English Micro Blog platform.

\section{Conclusion}

Micro Blog is a new communication way, which allows students to participate in other students' learning by reading, comment and discussion, and provide students with a platform for mutual 
exchange and cooperation. Therefore, they can solve the common problem with no limit of time and space This mobilizes students' learning enthusiasm greatly and expand the way of language input. As a useful supplement to classroom teaching, teachers' organization and guidance are necessary. The Micro Blog application should combine with classroom teaching to jointly promote the quality of college English teaching.

\section{References}

[1] Xue Fengnian. A Study on College English Teaching from the Perspective of Micro Media [J]. Theory and Practice of Education, 2016 (18):53-54.

[2] Sun Qian. A Study on the Application of Micro Blog in College English Reading Teaching [J]. College English (Academic Edition), 2015, (01): 46-49.

[3] Liu Dandan. A Study on College English Teaching Strategies Based on Micro Blog Platform [J]. Out School Education in China, 2014, (S3): 570-571.

[4] Wang Xiaohui. The Application and Research of Micro Blog in College English Writing Teaching [J]. Modern Communication, 2013, (09): 234.

[5] Xia Juan. An Analysis of the Application of Micro Blog in College English Teaching [J]. The Guide of Science and Education (Periodical Publication), 2013, (03): 142-143.

[6] He Ju. A Discussion on the Feasibility of the Application of Micro Blog in College English Teaching [J]. Overseas English, 2012, (16): 15-16.

[7] Diao Yefang. An Analysis of Application Advantages of Micro Blog in College English Teaching [J]. Qunwen World, 2012, (05): 188. 